\title{
ANÁlisis DE LA DISPOSICIÓN A PAGAR POR NO TRASBORDAR DE LOS USUARIOS DE TRANSPORTE PÚBLICO
}

\author{
RAQUEL ESPINO* \\ Departamento Análisis Económico Aplicado, UNIVERSIDAD DE LAS PALMAS DE GRAN CANARIA, \\ ESPAÑA \\ E-mail: raquel.espino@ulpgc.es
}

\section{RESUMEN}

La definición de políticas de transporte debe dirigirse a atender las necesidades de movilidad incidiendo en la importancia de ofrecer un servicio de transporte público que se adapte a su demanda. Dado que no siempre es posible atender dichas necesidades de movilidad con servicios directos, este trabajo plantea el análisis del trasbordo desde la perspectiva de los usuarios de transporte público en la isla de Gran Canaria (España). Para ello se realiza un experimento de Preferencias Declaradas que plantea diferentes situaciones de elección entre el servicio actual de bus con trasbordo y un servicio hipotético de bus directo. Con los resultados de las estimaciones, se obtiene la disposición a pagar por no trasbordar diferenciadas por tipo de trasbordo, siendo la disposición a pagar por evitar el trasbordo urbano menor que en el caso del trasbordo no-urbano.

Palabras clave: Preferencias Declaradas, disposición a pagar, modelos logit mixto, penalización del trasbordo

\section{Analysing the willingness to pay for not transfer of public transport users}

\begin{abstract}
The definition of public transport service should have to account the mobility necessity and try to adapt it to the transport demand. The transport supply cannot offer a direct service. In this work, we analysed the bus interchange from the users' subjective perceptions. For doing that, we designed a Stated Preference Experiment that present a choice between a current bus service and hypothetical direct bus service. The willingness to pay was obtained with the estimation results and the willingness to pay for not transfer defined by type of transfer, being lower the willingness to pay for not urban transfer.
\end{abstract}

Keywords: Stated Preference, willingness to pay, mixed logit models, transfer penalty

Clasificación JEL: R41, C25, C42, C81, C50 


\section{INTRODUCCIÓN}

El trasbordo es un elemento clave para la integración de las redes de transporte, resultando relevante en la definición del servicio de transporte público, dado que los usuarios deben invertir tiempo y dinero cuando no existe la posibilidad de llegar a su destino con un servicio directo de transporte público. La desutilidad percibida por el usuario de transporte público al realizar trasbordo se denomina penalización del trasbordo ${ }^{60}$ por algunos autores (Guo y Wilson, 2004; Currie, 2005). Iseki y Taylor (2009) profundizan en la definición de la penalización del trasbordo considerando la diferenciación de tres elementos claves: el tiempo de caminata hasta la siguiente parada, el tiempo de espera por el siguiente vehículo u otro modo y la penalización que supone en términos de molestias por la necesidad de trasbordar, lo que los autores denominan penalización ${ }^{61}$ pura del trasbordo. Esta penalización pura del trasbordo es independiente de los tiempos de caminata y de espera para realizar el trasbordo y se puede definir como la desutilidad que genera al usuario de transporte público cambiar de modo de transporte o de vehículo para llegar a su destino final.

Chowdhury y Ceder (2016) señalan que el trasbordo es un elemento crucial de los sistemas integrados de transporte. Por un lado, porque aumentan la accesibilidad a un mayor número de destinos $\mathrm{y}$, por otro lado, porque la fiabilidad del sistema integrado de transporte depende de un adecuado diseño de los puntos de intercambio para optimizar los trasbordos. Por su parte, Gschwender et al. (2016) demuestran que una configuración de la red donde las líneas secundarias alimentan a una línea troncal o principal puede ofertar un servicio de transporte más eficiente debido a la existencia de economías de densidad. Por tanto, los sistemas integrados de transporte tienden a una configuración donde la realización de uno o más trasbordos es necesario para garantizar que la cobertura de la red llegue a la mayoría de la población, estando dichos trasbordos coordinados para que los tiempos y costes invertidos por los usuarios sean los menores posibles. Un buen ejemplo de esta tendencia es la experiencia de reorganización del transporte público en Santiago de Chile (Navarrete y Ortúzar, 2013).

Sin embargo, a pesar de sus ventajas para ofertar un servicio de transporte público, el trasbordo constituye uno de los elementos más penalizados del viaje, no sólo por el incremento en el tiempo total del desplazamiento, sino también por la molestia asociada al cambio de vehículo y/o modo de transporte (Schakenbos et al., 2016). En este sentido, la percepción de los usuarios de transporte público de realizar trasbordo es negativa sin considerar los otros dos elementos relacionados con el trasbordo anteriormente comentados (Paulley et al., 2016).

Ante este contexto, la cuantificación de las preferencias de los viajeros respecto a los principales elementos que definen el viaje resulta esencial para evaluar aquellas políticas relacionadas con la reestructuración de la red. La necesidad de coordinar los horarios, junto con la necesidad de determinar la localización óptima de las estaciones de trasbordo resulta esencial a la hora de garantizar la movilidad y alcanzar los mayores beneficios sociales.

La mayoría de los estudios se centran en estudiar las valoraciones de los tiempos de viaje donde la literatura es muy amplia (Matas, 1991; Barrios y Martínez, 1999; Amador et al., 2005; Amador y González, 2005; Asensio y Matas, 2006; Cantos y Álvarez, 2006; Espino et al. 2006; Espino et al., 2007; Dell'Olio et al., 2011; Román et al., 2014a; entre otros ${ }^{62}$ ). Con respecto al estudio de la penalización pura del trasbordo destacan los trabajos de Iseki y Taylor (2009), Douglas y Jones (2013), Navarrete y Ortúzar (2013), Cascajo et al., (2018) y García-Martínez et al. (2018), siendo las dos últimas referencias sobre estudios realizados en España.

Este trabajo se centra en estudiar la valoración de la penalización pura del trasbordo como parte de un estudio más amplio de valoración global de los usuarios de transporte público de la isla de Gran Canaria a petición de la institución pública responsable (para más detalle ver Román et al., 2014b). Los resultados del estudio se consideran como dato para la valoración de los beneficios de los usuarios de

\footnotetext{
${ }^{60}$ Transfer penalty en inglés.

${ }^{61}$ Pure transfer penalty en inglés.

${ }^{62} \mathrm{Al}$ ser tan amplia la literatura en este tema se destacan los realizados en España y/o en transporte terrestre urbano e interurbano.
} 
transporte público ante una política de despenalización del trasbordo y contribuir al fomento del transporte público en la isla.

Para ello se realiza un experimento de Preferencias Declaradas (PD) donde se plantea a los usuarios que realizan trasbordo una situación de elección entre el servicio actual de bus, con trasbordo, y un servicio hipotético de bus directo. Se estiman modelos de elección discreta que permitan establecer las ponderaciones de los diferentes atributos considerados. Con estos resultados se calcula la valoración económica -en términos de disposición a pagar- por parte los usuarios de transporte público por no trasbordar.

En el caso de la isla de Gran Canaria es importante señalar que en el momento de realizar el estudio no existía ninguna integración tarifaria que permitiera el trasbordo entre las diferentes líneas de las empresas que ofertan dicho servicio en la isla ni tampoco una definición de los servicios que redujera los tiempos de trasbordo. Ante esta situación, surge la necesidad de estudiar las preferencias de los usuarios de transporte público que realizan trasbordo para valorar la penalización pura del trasbordo, esto es, cuánto estarían dispuestos a pagar por no trasbordar.

El resto del artículo se estructura de la siguiente manera. En la segunda sección se presentan el contexto del estudio, el experimento de PD realizado, así como la encuesta realizada y el análisis descriptivo de la muestra. En la tercera sección se muestran los resultados de los modelos estimados. Las disposiciones a pagar por no trasbordar se presentan en la cuarta sección. En la quinta y última sección se exponen las principales conclusiones que se derivan del estudio.

\section{DATOS}

\subsection{Contexto del estudio}

El estudio se realiza en la isla de Gran Canaria (España). Gran Canaria tiene 845.195 habitantes, mientras que la capital de la isla, Las Palmas de Gran Canaria, tiene 383.308 habitantes, un $45 \%$ del total de la población de la isla. La densidad de población es una de las más altas del archipiélago de Canarias con unos 542 habitantes por $\mathrm{km} 2$, siendo esta densidad de población casi 7 veces mayor en el en el caso de la ciudad de Las Palmas de Gran Canaria, esto es, 3.812 habitantes por km (ISTAC, 2016).

El servicio regular de transporte de pasajeros en la isla es ofrecido por dos empresas: Guaguas Municipales y Global. La empresa Guaguas Municipales tiene la concesión de los servicios de transporte urbano en la capital de la isla dando servicio de transporte público urbano entre los distintos puntos de la capital. Este servicio de transporte público urbano tiene una tarifa plana con un coste por viaje independiente de la distancia recorrida. La empresa Guaguas Municipales oferta diferentes títulos $^{63}$ de viaje -además del billete sencillo- que suponen diferentes descuentos sobre la tarifa plana.

La empresa Global tiene la concesión del transporte regular de transporte interurbano entre la capital y los otros 20 municipios de la isla, además de los núcleos turísticos y un servicio al aeropuerto ${ }^{64} \mathrm{de}$ Gran Canaria. La tarifa está definida en función del número de kilómetros recorridos con un mínimo de percepción definido para los viajes inferiores a 13 kilómetros. La empresa Global oferta diferentes títulos ${ }^{65}$ de viaje que suponen diferentes descuentos sobre la tarifa base.

Para este trabajo se considera como población objeto de estudio a los usuarios del transporte público en la isla de Gran Canaria. En este tipo de estudios la población relevante son aquellos usuarios del transporte púbico que estén familiarizados con el trasbordo, esto es, que realicen con cierta regularidad trasbordo en sus desplazamientos (Cascajo et al., 2018; García-Martínez et al., 2018; Dell'Olio et al., 2011; Wardman y Hine, 2000).

Por tanto, la encuesta se realiza tanto a los usuarios del transporte urbano como interurbano que realizan un trasbordo entre cualquiera de las dos empresas de transporte regular de viajeros: Guaguas

\footnotetext{
${ }^{63}$ El título de viaje más utilizado según datos de la empresa Guaguas Municipales es el bono guagua que tiene un descuento del $40 \%$ sobre la tarifa del billete sencillo.

${ }^{64}$ Un servicio desde la ciudad de Las Palmas de Gran Canaria al aeropuerto y otro servicio desde Maspalomas, principal núcleo turístico, al aeropuerto.

${ }^{65}$ El título de viaje mas utilizado según datos de la empresa Global es la tarjeta insular con un descuento variable del 30-50\% de la tarifa base en función del uso de la tarjeta.
} 
Municipales y Global. Así tenemos tres posibles situaciones de trasbordo; el trasbordo urbano cuando se realiza entre líneas de Guaguas Municipales. El trasbordo interurbano cuando se realiza entre líneas de Global y finalmente el trasbordo interurbano-urbano cuando se realiza entre una línea de Global y una línea de Guaguas Municipales.

Por tanto, el estudio se centra en el trasbordo entre las diferentes líneas de bus existentes y ofertadas por las dos empresas de transporte regular de viajeros. Estos trasbordos implican solamente un cambio de vehículo y se realiza en las paradas de bus ${ }^{66}$, en las dos estaciones ${ }^{67}$ de buses o en los cuatro puntos de intercambio ${ }^{68}$ existentes. El trasbordo se realiza en el mismo nivel sin acceso vía escaleras o ascensores y el tiempo de caminata puede considerarse cero dado que - en la mayoría de los casos - los individuos no necesitan caminar para acceder al otro bus.

En cuanto al servicio de transporte hay una clara diferencia entre el servicio de transporte urbano y el servicio de transporte interurbano. El servicio de transporte urbano presenta unas frecuencias mayores, esto es, más buses a la hora o menor tiempo entre dos buses consecutivos que en el caso del transporte interurbano donde las frecuencias son menores. El tiempo de espera medio se establece que es la mitad de la frecuencia del bus, esto es, la mitad del tiempo entre dos buses consecutivos (Osuna y Newell, 1972). Los usuarios programan su salida en función de esta información para reducir los tiempos de espera. A pesar de que el transporte interurbano tiene unas frecuencias menores, la puntualidad de este tipo de servicio de buses es mayor que en el caso del transporte urbano, donde la fiabilidad del servicio es menor dado que no existe una red de carriles buses ni preferencia semafórica con lo que los servicios de bus pueden sufrir retrasos y no cumplir con los tiempos de viaje establecidos.

Por otra parte, los tiempos de viaje en transporte interurbano son, en general, mayores dado que la distancia recorrida es mayor que en el caso del transporte urbano al ser un servicio entre los distintos municipios de la isla y la capital. Además, los trasbordos en el caso del transporte urbano se realizan bien en las paradas de bus o bien en los cuatro puntos de intercambios comentados anteriormente. En el caso del transporte interurbano, la mayoría de los trasbordos se realizan en las dos estaciones de buses. Ante estas diferencias en el servicio de transporte urbano e interurbano puede considerarse que haya diferentes preferencias de los usuarios según el tipo de trasbordo. Para más información sobre la oferta de transporte público en Gran Canaria se puede consultar Espino (2003).

Ante este contexto se realiza una encuesta constituida por tres bloques de preguntas. El primero recoge la información relativa al viaje necesaria para adaptar el experimento de PD. El segundo está formado por el experimento de PD que enfrenta al individuo a la elección entre el servicio actual con trasbordo y un servicio hipotético de bus directo definido a partir de una combinación de los atributos más relevantes y adaptado a la información de cada entrevistado (bloque 1 de preguntas). Finalmente, el tercer bloque recoge la información socioeconómica a nivel de hogar y a nivel del individuo.

\subsection{Experimento de Preferencias Declaradas}

El segundo bloque de la encuesta lo constituye el experimento de PD donde se presenta al entrevistado un juego de elección entre dos alternativas de transporte: la alternativa actual de bus con trasbordo y un servicio de bus directo adaptado a la situación de cada individuo.

Para la definición de los atributos y sus niveles se realiza un grupo focal con 8 personas usuarias de transporte público que realizan alguno de los tres tipos de trasbordos posibles. El desarrollo del grupo focal tiene en cuenta la información obtenida en el estudio previo realizado sobre los índices de calidad del transporte público (Román et al., 2014b) así como la información facilitada por las empresas de transporte público que ofrecen el servicio regular de transporte de pasajeros en la isla de Gran Canaria. En el estudio previo se estudiaron diferentes atributos sobre la calidad del servicio de transporte público, incluyéndose además de los atributos modales de tiempo de viaje, coste de viaje y frecuencia, otras variables como información disponible en las paradas de buses, existencia o no de marquesinas en las

\footnotetext{
${ }^{66}$ La distancia media entre paradas en la ciudad de Las Palmas de Gran Canaria es de 0,5 km.

${ }^{67}$ Estación de Guaguas de San Telmo e Intercambiador de Santa Catalina.

${ }^{68}$ Los cuatro puntos de intercambio son: Teatro, Mercado de Vegueta, Alameda de Colón y Parque de Santa Catalina.
} 
paradas de bus, tipo de conducción del chófer y nivel de limpieza del bus. Para más detalle revisar Román et al. (2014b).

Del grupo focal se obtuvieron las variables a definir en el escenario para el servicio de bus directo. Dado que dicho grupo focal se centra en el servicio de bus directo, diferentes variables relevantes en el contexto del trasbordo no resultan identificadas como variables en la definición de los escenarios como son la información disponible, la seguridad en los puntos de intercambio y las instalaciones para realizar los trasbordos, entre otras.

Respecto a otras variables que también afectan al trasbordo como la climatología (Cascajo et al., 2018, Iseki y Taylor, 2009) no es una variable que pueda influir en las preferencias de los usuarios dado que Canarias en general y Las Palmas de Gran Canaria en particular son conocidas por su buen clima durante todo el año. Otra variable considerada como aglomeración en el momento de realizar el trasbordo tampoco es una variable relevante en este estudio. La ciudad de Las Palmas de Gran Canaria es una ciudad de tamaño medio (inferior a 500.000 habitantes) y no presenta niveles críticos de aglomeración en la realización de los trasbordos. En España, el estudio realizado para Vitoria-Gasteiz por García-Martínez (2018) esta variable tampoco fue considerada. En sistemas de transporte público con mayor densidad podría ser una variable relevante como ocurre en el caso de Madrid (GarcíaMartínez, 2018; García-Martínez et al., 2018). Por último, la definición de diferentes niveles a la hora de realizar trasbordo y/o escaleras tampoco es una variable relevante dado que los trasbordos se realizan al mismo nivel en las paradas de bus, en las estaciones de bus o los cuatro puntos de intercambio anteriormente mencionado (Schakenbos et al., 2014; Raveau et al., 2013, Navarrete y Ortúzar, 2013; Douglas y Jones, 2013; Guo y Wilson, 2011; Wardman y Hine, 2000).

Por tanto, el juego de elección planteado se basa en un diseño experimental que considera sólo tres atributos para las dos alternativas presentadas al individuo: tiempo total del viaje, coste total del viaje y tiempo de servicio entre dos buses consecutivos. Cada atributo se define a dos niveles resultando un diseño ${ }^{69}$ factorial completo de ocho opciones (23). Al ser este número reducido, se presentan las ocho opciones de elección al individuo teniendo, por tanto, un diseño ortogonal que permite medir todas las interacciones entre los tres atributos considerados, así como el estudio de especificaciones no lineales. Para construir un diseño adaptado a la experiencia de cada persona entrevistada se utiliza el software WinMINT 2.1 (Rand Europe, 2001). Para ello la entrevista se realiza con ordenador portátil por entrevistadores instruidos previamente. La presentación de cada uno de los ocho escenarios de elección se realiza de manera aleatoria para cada uno de los individuos entrevistados para reducir los posibles sesgos (Bradley y Kroes, 1990; Espino et al., 2004).

Los atributos del servicio de bus con trasbordo son el tiempo de viaje, el coste del viaje y la frecuencia expresada como el tiempo entre dos buses consecutivos. El tiempo de viaje es la suma del tiempo de viaje del primer bus (T1), el tiempo de viaje del segundo bus (T2) y el tiempo de trasbordo (TT) como tiempo de espera hasta tomar el segundo bus. El coste del viaje es la suma del precio pagado en el primer bus (C1) y del precio pagado en el segundo bus (C2). La frecuencia es el tiempo entre dos buses consecutivos y se define como el valor máximo de la frecuencia del primer (F1) y del segundo bus (F2). En la

Tabla 1 se presentan los atributos del servicio actual de bus con trasbordo.

El servicio hipotético de bus directo se define tomando como referencia los datos del servicio de bus con trasbordo que realiza el entrevistado, cuyos datos son obtenidos en el bloque 1 de la encuesta, previo al experimento de PD. La definición de los niveles del servicio de bus directo se plantea con base a la compensación económica para disponer de un servicio directo que implique un menor tiempo suponen un coste mayor y por tanto una frecuencia menor (Douglas y Jones, 2013).

El tiempo de viaje para la alternativa de servicio de bus directo toma como referencia la suma de los dos tiempos de viaje del primer y segundo trayecto en bus $(\mathrm{T}=\mathrm{T} 1+\mathrm{T} 2)$ y se define como un $25 \%$ menor que este tiempo para el nivel 1 y un 50\% menor para el nivel 2. Por su parte, el coste del viaje toma como referencia el coste del viaje en servicio actual de bus con trasbordo $(\mathrm{C}=\mathrm{C} 1+\mathrm{C} 2)$, es el coste total declarado por el entrevistado, de manera que el coste del servicio de bus directo es un $25 \%$ y un $50 \%$ mayor que este valor para los niveles 1 y 2 , respectivamente. Finalmente, la frecuencia expresada como

\footnotetext{
${ }^{69} \mathrm{Al}$ tratarse de un diseño factorial completo no es necesario un diseño eficiente. Los diseños eficientes se recomiendan cuando se realiza un diseño factorial fraccional (Bliemer y Rose, 2005; Bliemer y Rose, 2010; Román et al., 2012).
} 

tiempo de servicio entre dos autobuses consecutivos, se define a partir del valor máximo de las frecuencias del primer y segundo trayecto realizado por el individuo, siendo definidos los niveles igual a este valor máximo incrementado en un $25 \%$ y en un $50 \%$ para el nivel 1 y 2 respectivamente. La Figura 4 muestra un ejemplo de la pantalla mostrada al individuo en una situación de elección.

Tabla 1

Atributos del servicio de bus con trasbordo

\begin{tabular}{|c|c|c|}
\hline Atributos & \multicolumn{2}{|l|}{ Actual Servicio de Bus } \\
\hline \multirow{3}{*}{$\begin{array}{l}\text { Tiempo de viaje } \\
\text { (minutos) }\end{array}$} & Tiempo de viaje del primer bus $\left(T_{1}\right)$ & \multirow[t]{3}{*}{$\mathrm{T}_{1}+\mathrm{T}_{2}+\mathrm{TT}$} \\
\hline & Tiempo de viaje del segundo bus $\left(T_{2}\right)$ & \\
\hline & Tiempo de trasbordo (TT) & \\
\hline \multirow{2}{*}{$\begin{array}{l}\text { Coste } \\
\text { (euros) }\end{array}$} & Coste del primer bus $\left(\mathrm{C}_{1}\right)$ & \multirow[t]{2}{*}{$\mathrm{C}=\mathrm{C}_{1}+\mathrm{C}_{2}$} \\
\hline & Coste del segundo bus $\left(\mathrm{C}_{2}\right)$ & \\
\hline \multirow{2}{*}{$\begin{array}{c}\text { Frecuencia } \\
\text { (minutos) }\end{array}$} & Tiempo entre dos buses consecutivos $-1^{\circ}$ bus $\left(F_{1}\right)$ & \multirow{2}{*}{$\begin{array}{c}\mathrm{F}=\text { Máximo } \\
\left(\mathrm{F}_{1}, \mathrm{~F}_{2}\right)\end{array}$} \\
\hline & Tiempo entre dos buses consecutivos $-2^{\circ}$ bus $\left(F_{2}\right)$ & \\
\hline
\end{tabular}

En la ¡Error! La autoreferencia al marcador no es válida. se presentan los atributos y niveles utilizados en el experimento de PD para cada alternativa (Louviere et al., 2000; Espino et al., 2004).

Tabla 2

Atributos y niveles del experimento de $P D$

\begin{tabular}{|c|c|c|c|}
\hline Atributos & Niveles & $\begin{array}{c}\text { Servicio de Bus con } \\
\text { trasbordo }\end{array}$ & Servicio de Bus Directo \\
\hline \multirow{4}{*}{$\begin{array}{c}\text { Tiempo de viaje } \\
\text { (minutos) }\end{array}$} & Base & $\mathrm{T}_{1}+\mathrm{T}_{2}+\mathrm{TT}$ & - \\
\cline { 2 - 4 } & 2 & & $0.75 \cdot\left(\mathrm{T}_{1}+\mathrm{T}_{2}\right)$ \\
\cline { 2 - 4 } & Base & $\mathrm{C}=\mathrm{C}_{1}+\mathrm{C}_{2}$ & $0.5 \cdot\left(\mathrm{T}_{1}+\mathrm{T}_{2}\right)$ \\
\cline { 2 - 4 } & 1 & - & - \\
\cline { 2 - 4 } Coste (euros) & 2 & - & $1.25 \cdot \mathrm{C}$ \\
\hline \multirow{3}{*}{$\begin{array}{c}\text { Frecuencia } \\
\text { (minutos) }\end{array}$} & 1 & $\mathrm{~F}=$ Máximo $\left(\mathrm{F}_{1}, \mathrm{~F}_{2}\right)$ & $1.50 \cdot \mathrm{C}$ \\
\cline { 2 - 4 } & 2 & - & $1.25 \cdot F$ \\
\cline { 2 - 4 } & & - & $1.50 \cdot F$ \\
\hline
\end{tabular}


Figura 4

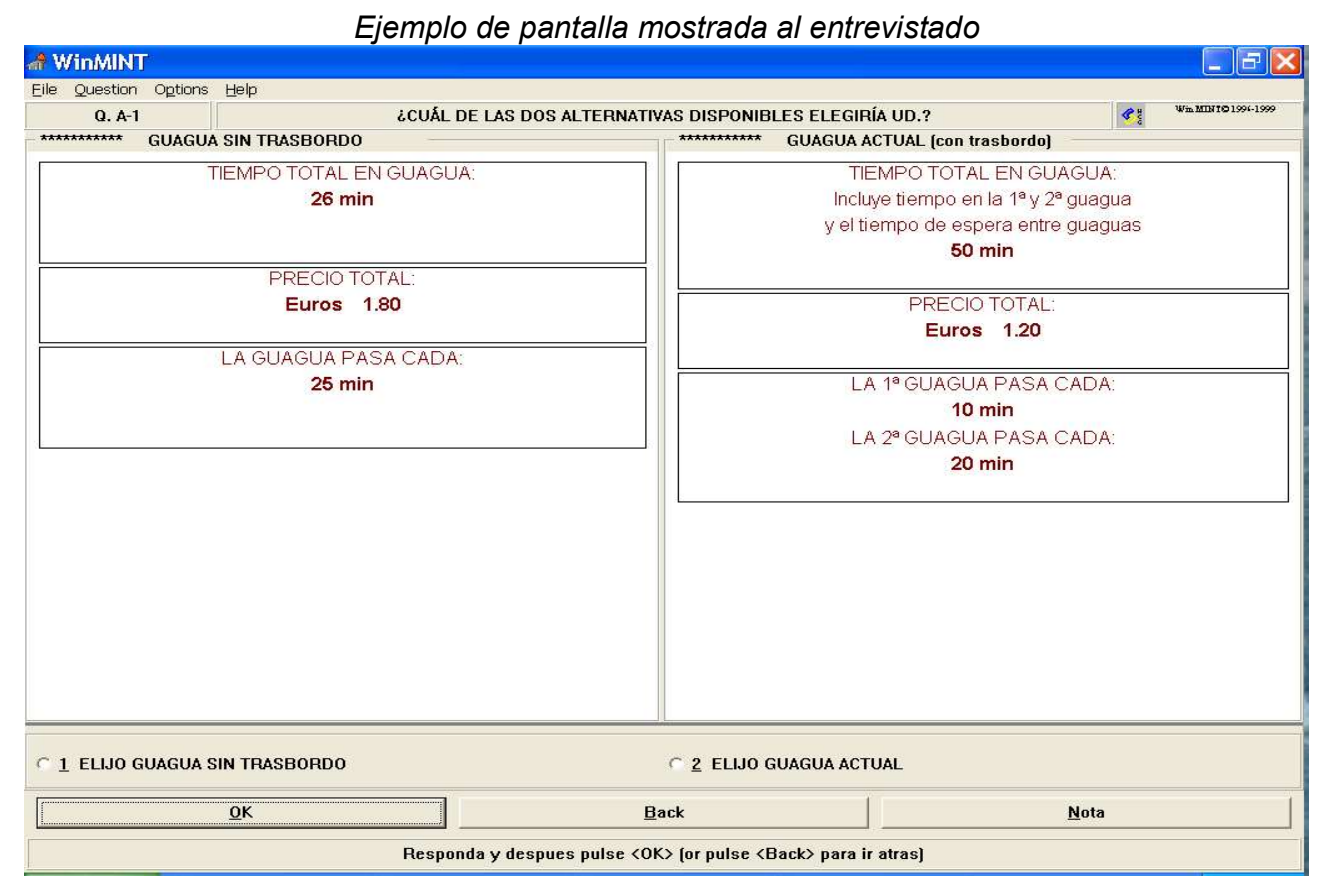

\subsection{Descripción de la muestra}

La encuesta realizada es una encuesta $a d-h o c$ que pretende obtener información sobre la percepción del trasbordo por parte de los usuarios de transporte público en Gran Canaria. Las empresas de transporte regular de viajeros no disponen de información sobre el perfil del usuario de transporte público que realiza trasbordo en el momento del estudio. Para obtener una muestra representativa se realiza un muestreo aleatorio simple al $90 \%$ de nivel de confianza ante una población superior a 100.000 individuos y un error muestral del 5\% se necesitan realizar unas 270 encuestas. Por este motivo, se proponen realizar 300 encuestas, 100 encuestas por cada tipo de trasbordo. Por otro lado, para seleccionar a los diferentes usuarios de transporte público que realizan trasbordo en toda la red de transporte se realiza un análisis de clúster de las diferentes líneas y se seleccionan aquellas representativas de cada clúster. Los pasajeros se seleccionan aleatoriamente en las dos estaciones de buses $^{70}$ y en los cuatro puntos de intercambio ${ }^{71}$ a propuesta de las empresas de transporte siempre y cuando que realicen algún tipo de trasbordo en alguna de las líneas seleccionadas.

Finalmente, se realizaron un total de 302 encuestas durante el mes de Octubre de 2005. Por tipo de trasbordo, 102 encuestas fueron entre líneas de Guaguas Municipales (trasbordo urbano), 99 entre líneas de Global (trasbordo interurbano) y 101 entre líneas de Guaguas Municipales y Global (trasbordo interurbano-urbano). Cada entrevistado responde a 8 opciones de elección entre su servicio actual de bus con trasbordo y el servicio de bus directo propuesto, siendo la base de datos de un total de 302 individuos y 2416 observaciones.

El análisis descriptivo de la muestra se presenta Tabla 3. Se observa que hay mayor número de usuarios mujeres con un 57\% del total de la muestra. La edad media es de 36 años, destacando que la población joven es un porcentaje relevante de la muestra con un 53\% de usuarios entre 18-30 años. Las personas mayores de 65 años son solo un $8,6 \%$ de la muestra, lo que está en línea con los estudios realizados que indican que las personas mayores están menos dispuestas a realizar viajes en transporte público con trasbordo (Cascajo et al., 2018; Wardman y Hine, 2000). En cuanto al motivo de viaje, más del $52 \%$ de la muestra realiza el viaje por motivo obligatorio, esto es, un $26,49 \%$ por trabajo o negocios y un $26,16 \%$ por estudios. En relación con el nivel de estudios, sólo un $25 \%$ de los viajeros encuestados tiene estudios medios o superiores frente a un $54 \%$ que declara tener estudios secundarios. Respecto a la frecuencia con la que utilizan el transporte público, en general, son usuarios frecuentes con más de

\footnotetext{
${ }^{70}$ Estación de Guaguas de San Telmo e Intercambiador de Santa Catalina.

${ }^{71}$ Los cuatro puntos de intercambio son: Teatro, Mercado de Vegueta, Alameda de Colón y Parque de Santa Catalina.
} 

un $50 \%$ de la muestra que realiza el viaje más 5 de veces por semana. Por tanto, los entrevistados son usuarios conocedores del sistema de transporte público.

Tabla 3

Análisis descriptivo de la muestra

\begin{tabular}{|c|c|c|c|}
\hline \multicolumn{2}{|c|}{ Característica Socioeconómica } & \multicolumn{2}{|c|}{ Total } \\
\hline \multirow[t]{2}{*}{ Sexo: } & Hombres & 130 & $43,05 \%$ \\
\hline & Mujeres & 172 & $56,95 \%$ \\
\hline \multirow[t]{4}{*}{ Edad: } & & $36^{*}$ & $16,52^{* *}$ \\
\hline & 18-30 años & 160 & $53 \%$ \\
\hline & 31-65 años & 116 & $38,4 \%$ \\
\hline & Mayor de 65 años & 26 & $8,6 \%$ \\
\hline \multirow[t]{7}{*}{ Motivo del viaje: } & Trabajo & 80 & $26,49 \%$ \\
\hline & Estudios & 79 & $26,16 \%$ \\
\hline & Visita a un familiar & 48 & $15,89 \%$ \\
\hline & Por ocio/turismo & 42 & $13,9 \%$ \\
\hline & Por visita médica & 24 & $7,9 \%$ \\
\hline & Por compras & 15 & $4,96 \%$ \\
\hline & Otros & 15 & $4,96 \%$ \\
\hline \multirow[t]{3}{*}{ Frecuencia del viaje: } & $1-5$ viajes/semana & 145 & $48,01 \%$ \\
\hline & $6-10$ viajes/semana & 124 & $41,05 \%$ \\
\hline & Más 10 viajes/semana & 33 & $10,93 \%$ \\
\hline \multirow[t]{4}{*}{ Nivel educativo: } & Sin estudios & 6 & $2 \%$ \\
\hline & Estudios primarios & 57 & $18,8 \%$ \\
\hline & Estudios secundarios & 163 & $54 \%$ \\
\hline & Con estudios universitarios & 78 & $25,2 \%$ \\
\hline
\end{tabular}

* Media ** Desviación típica

\section{MODELIZACIÓN Y RESULTADOS DE LAS ESTIMACIONES}

La microeconomía de las elecciones discretas es la teoría que fundamenta los modelos de demanda desagregados siendo la teoría de la utilidad aleatoria el marco teórico para la especificación econométrica de estos modelos (Domencich y McFadden 1975; McFadden, 1981; Ortúzar y Willumsen, 2011). Esta teoría propone que cada individuo q asocia a cada alternativa $\mathrm{j}$ una utilidad. Esta utilidad asociada a cada alternativa es de tipo estocástico (Ujq), siendo elegida aquella alternativa que maximiza su utilidad. Esta utilidad está definida por la siguiente expresión:

$$
U_{j q}=V_{j q}+\varepsilon_{j q}
$$

donde Vjq es la utilidad representativa o sistemática y ejq es el término de error que incluye los efectos no observados por el investigador. La utilidad representativa ${ }^{72}$ Vjq está determinada por los atributos observables de la alternativa y de las características socioeconómicas del individuo. El término de error ejq es una variable aleatoria con media cero y una determinada distribución. La distribución del término de error define los diferentes tipos de modelos desagregados de demanda o de elección discreta. En este trabajo se han estimado modelos Logit Mixto (ML) con componentes de error que permiten considerar correlación entre las observaciones de un mismo individuo (Train, 2009). Este modelo es muy útil en el caso de datos de PD donde cada individuo responde a un mismo número de situaciones de elección. Este modelo permite recoger la potencial correlación entre las respuestas de dicho individuo (McFadden y Train, 2000). La especificación del modelo $\mathrm{ML}^{73}$ con componentes de error se especifica de la siguiente manera:

\footnotetext{
${ }^{72}$ Esta es la utilidad que se estima con los modelos de elección discreta.

${ }^{73}$ Para más detalle consultar Ortúzar y Willumsen (2011) y Train (2009).
} 


$$
U_{j q}=\theta^{\prime} x_{j q}+u_{q}^{\prime} z_{j q}+\varepsilon_{j q}
$$

donde:

xjq es un vector de atributos observados de la alternativa $\mathrm{j}$ para el individuo $\mathrm{q}$

zjq son vectores de atributos observados de la alternativa $\mathrm{j}$ para el individuo q. Es el término del componente de error que, junto con $\varepsilon j q$, define la parte estocástica del modelo. Si es idénticamente igual a 0 indica que no existe correlación entre la utilidad de las alternativas y el modelo equivale a un modelo Logit Multinomial.

$\theta$ es un vector de coeficientes definido para cada atributo observado xjq, que se consideran constantes para cada uno de los individuos y puede variar entre alternativas

uq es un vector de términos aleatorios con media 0 y covarianza $\mathrm{W}$

عjq es el término de error que distribuye idéntica e independientemente Gumbel.

La parte estocástica o no observada de la función de utilidad en un modelo ML con componentes de error se puede especificar como:

$$
\eta_{j q}=u_{q}^{\prime} z_{j q}+\varepsilon_{j q}
$$

La definición de componentes de error distintos de cero entre alternativas determina cierta correlación entre las utilidades de las alternativas de manera que:

$$
\operatorname{Cov}\left(\eta_{j q}, \eta_{i q}\right)=\mathrm{E}\left(u_{q}^{\prime} z_{j q}+\varepsilon_{j q}\right)\left(u_{q}^{\prime} z_{i q}+\varepsilon_{i q}\right)=z_{j q} W z_{i q}
$$

siendo W la covarianza de $\mu \mathrm{q}$ y distribuyendo idéntica e independientemente como una distribución normal con media cero y desviación típica oq. Para más detalles sobre el modelo ML con componentes de error se puede consultar Train (2009) y Revelt y Train (1998).

Se estiman modelos ML con componentes de error homocedástico74, esto es, que se considera correlación entre las observaciones de un mismo individuo y que dicho componente de error es el mismo para las dos alternativas de elección. Los modelos se estimaron con el software Biogeme 2.4 (Bierlaire, 2003).

Además, se probaron diferentes especificaciones para la función de utilidad representativa (V). En primer lugar, se prueba una especificación lineal para los parámetros de los tres atributos considerados (tiempo de viaje, coste de viaje y frecuencia) y constante modal para la alternativa de servicio actual de bus con trasbordo (modelo 1). En segundo lugar, se prueban modelos que incluyen la interacción entre los atributos considerados en el experimento de PD, esto es, tres interacciones: tiempo con coste, tiempo con frecuencia y coste con frecuencia. Ninguna de las interacciones estudiadas resulta estadísticamente significativa. En tercer lugar, se estudia una especificación que considera el tipo de trasbordo en la constante modal (modelo 2 y modelo 3 ) que resultan estadísticamente significativas. En tercer lugar, se estudia la variación sistemática en los gustos mediante la definición de variables socioeconómicas interactuando con los atributos modales (Espino et al. 2006; Espino et al., 2007). Las variables socioeconómicas consideradas son: género, edad y motivo del viaje. En cuarto lugar, se estudia la variación aleatoria en los gustos definiendo parámetros aleatorios para los atributos modales y finalmente se estudian modelos que combinan variación sistemática y aleatoria en los gustos. El estudio de la variación sistemática y aleatoria en los gustos se realiza tanto para la especificación del modelo 1 como para la especificación del modelo 2, sin obtener estadísticamente mejores resultados. Por este motivo, sólo se presentan los resultados de los modelos anteriormente mencionados: modelo 1 y modelo 2. Además, se estima un tercer modelo (modelo 3) que permite comprobar, de acuerdo con el principio de parsimonia, si existen diferencias estadísticamente significativas entre la penalización del trasbordo interurbano (Oti) y la penalización del trasbordo interurbano-urbano (Otiu), penalización estudiada mediante la especificación de constantes modales específicas por tipo de trasbordo en la alternativa de servicio actual de bus con trasbordo.

\footnotetext{
${ }^{74}$ También se estiman modelos ML con componentes de error heterocedásticos y no resultan significativos estadísticamente.
} 
La especificación de la función de la utilidad representativa de cada alternativa para el modelo 1 es la siguiente:

$$
\begin{gathered}
V_{S D}=\theta_{t} \cdot T+\theta_{c} \cdot C+\theta_{F} \cdot F+\sigma \\
V_{S T}=\theta_{S T}+\theta_{t} \cdot T+\theta_{c} \cdot C+\theta_{F} \cdot F+\sigma
\end{gathered}
$$

donde:

VSD es la utilidad representativa de la alternativa de servicio de bus directo

VST es la utilidad representativa de la alternativa de servicio actual de bus con trasbordo

$\theta \mathrm{ST}$ es la constante modal de la alternativa de servicio actual de bus con trasbordo para los tres tipos de trasbordo

$\theta t$ es el parámetro para el tiempo de viaje

$\theta \mathrm{c}$ es el parámetro para el coste de viaje

$\theta \mathrm{F}$ es el parámetro para la frecuencia

$\sigma$ es la desviación típica ${ }^{75}$ del componente de error homocedástico que tiene en cuenta la correlación entre las observaciones de un mismo individuo

Para el modelo 2, se considera la misma especificación lineal en los atributos modales y la constante modal se especifica interactuando con una variable dummy para cada tipo de trasbordo con el objetivo de estudiar si existe diferente valoración de la penalización del trasbordo por cada uno de los tres tipos de trasbordo posibles (urbano, interurbano, interurbano-urbano). La especificación de las funciones de utilidad estimadas para las dos alternativas para el modelo 2 son las siguientes:

$$
\begin{gathered}
V_{S D}=\theta_{t} \cdot T+\theta_{c} \cdot C+\theta_{F} \cdot F+\sigma \\
V_{S T}=\theta_{t u} \cdot D_{t u}+\theta_{t i} \cdot D_{t i}+\theta_{t i u} \cdot D_{t i u}+\theta_{t} \cdot T+\theta_{c} \cdot C+\theta_{F} \cdot F+\sigma
\end{gathered}
$$

donde:

VSD es la utilidad representativa de la alternativa de servicio de bus directo

VST es la utilidad representativa de la alternativa de servicio actual de bus con trasbordo

$\theta$ tu es la constante modal de la alternativa de servicio actual de bus con trasbordo para trasbordo urbano

$\theta$ ti es la constante modal de la alternativa de servicio actual de bus con trasbordo para trasbordo interurbano

Otiu es la constante modal de la alternativa de servicio actual de bus con trasbordo para trasbordo interurbano-urbano

$\theta t$ es el parámetro para el tiempo de viaje

$\theta \mathrm{c}$ es el parámetro para el coste de viaje

$\theta \mathrm{F}$ es el parámetro para la frecuencia

$\sigma$ es la desviación típica del componente de error homocedástico que tiene en cuenta la correlación entre las observaciones de un mismo individuo.

Para el modelo 3 solo se consideran dos tipos de trasbordo: urbano y no urbano. El trasbordo urbano es cuando los usuarios realizan trasbordo entre las líneas de Guaguas Municipales (como en el modelo 2) y el trasbordo no urbano es cuando el trasbordo se realiza entre líneas de Global (trasbordo interurbano) y cuando se realiza entre líneas de Global y de Guaguas Municipales (trasbordo interurbano-urbano). La especificación de este modelo 3 es una versión restringida del modelo 2 . La especificación de las funciones de utilidad estimadas para las dos alternativas para el modelo 3 son las siguientes:

$$
V_{S D}=\theta_{t} \cdot T+\theta_{c} \cdot C+\theta_{F} \cdot F+\sigma
$$

\footnotetext{
${ }^{75}$ Se define el componente de error con una variable que distribuye idéntica e independientemente normal con media cero y desviación típica $\sigma$.
} 


$$
V_{S T}=\theta_{t u} \cdot D_{t u}+\theta_{t n u} \cdot D_{t n u}+\theta_{t} \cdot T+\theta_{c} \cdot C+\theta_{F} \cdot F+\sigma
$$

donde:

VSD es la utilidad representativa de la alternativa de servicio de bus directo

VST es la utilidad representativa de la alternativa de servicio actual de bus con trasbordo

$\theta$ tu es la constante modal de la alternativa de servicio actual de bus con trasbordo para trasbordo urbano

$\theta$ tnu es la constante modal de la alternativa de servicio actual de bus con trasbordo para trasbordo no-urbano

$\theta t$ es el parámetro para el tiempo de viaje

$\theta \mathrm{c}$ es el parámetro para el coste de viaje

$\theta \mathrm{F}$ es el parámetro para la frecuencia

$\sigma$ es la desviación típica del componente de error homocedástico que tiene en cuenta la correlación entre las observaciones de un mismo individuo.

Los parámetros estimados $(\theta)$ miden la percepción de los diferentes atributos de los individuos, esto es, la utilidad marginal ${ }^{76}$ de cada uno de los atributos dentro de la función de utilidad representativa $(\mathrm{V})$.

Los atributos de las dos alternativas considerados en la estimación de los modelos son el tiempo de viaje (T), el coste del viaje (C) y la frecuencia (F). También se definen variables ficticias para definir los diferentes tipos de trasbordo considerados en la estimación. La definición de las variables consideradas en la estimación, así como los signos esperados de los parámetros de estas variables se presentan en la Tabla 4.

En la Los modelos 1 y 3 son una versión restringida del modelo 2 que establece constantes modales específicas por tipo de trasbordo (urbano, interurbano e interurbano-urbano), mientras que el modelo 1 es una versión restringida del modelo 3 que especifica constantes modales según el trasbordo sea urbano o no-urbano. Para poder determinar el mejor modelo en términos estadísticos se utiliza el test de razón de verosimilitud (Ortúzar y Willumsen, 2011), que establece como hipótesis nula que el modelo restringido es una especificación correcta con respecto al modelo no restringido. Dicho test tiene la siguiente expresión:

$$
L R=-2\left\{l^{*}\left(\hat{\theta}_{r}\right)-l^{*}(\hat{\theta})\right\}
$$

donde $l^{*}\left(\hat{\theta}_{r}\right)$ es la log-verosimilitud en convergencia del modelo restringido y $l^{*}(\hat{\theta})$ es la del modelo más general. Este test estadístico distribuye asintóticamente $\chi 2$ con $\mathrm{r}$ grados de libertad, siendo $\mathrm{r}$ el número de restricciones lineales. La hipótesis nula se rechaza si $L R>X_{r, 95}^{2}$, por lo que el modelo general es aceptado. El modelo 1 es una versión restringida del modelo 2 con tres restricciones lineales, así el valor de LR es 11,4>7,82, así que se acepta el modelo 2, donde la constante modal se especifica por tipo de trasbordo. El modelo 3 es una versión restringida del modelo 2 con dos restricciones lineales, el valor de LR es $0,39<7,82$, por lo que se acepta la hipótesis nula, esto es, el modelo 3 es una especificación correcta. Si se comparan el modelo 1 como modelo restringido del modelo 3 con dos restricciones lineales se obtiene un valor de LR igual a 11,01>5,99 por lo que se rechaza la hipótesis nula y el modelo 3 es una especificación correcta. Por tanto, según el test de razón de verosimilitud y según los contrastes de hipótesis realizados, el modelo 3 con dos parámetros específicos para la constante modal es una mejor especificación en términos estadísticos que los modelos 1 y 2.

Tabla 5 se presentan los resultados de los tres modelos estimados que son estadísticamente mejores. Todos los parámetros resultan con el signo esperado y son estadísticamente significativos al $95 \%$ de nivel de confianza. En los dos modelos existe correlación entre observaciones dado que el valor de sigma $(\sigma)$ resulta diferente de cero y estadísticamente significativo al $95 \%$ de nivel de confianza, lo que indica que existe correlación entre las observaciones de un mismo individuo. Este resultado es el

\footnotetext{
${ }^{76}$ La utilidad marginal es la derivada parcial de la función de utilidad que se estima y en el caso de una especificación lineal, esta utilidad marginal es igual al parámetro estimado para el atributo modal correspondiente.
} 

esperado en el caso de datos de PD y la consideración de dicha correlación es la práctica adecuada (Louviere et al., 2000; Train, 2009).

Para los tres modelos se obtiene que la utilidad marginal de la frecuencia es mayor que la utilidad marginal del tiempo de viaje, esto es, que el aumento de un minuto del tiempo entre dos buses consecutivos genera mayor desutilidad que el aumento de un minuto del tiempo de viaje. Este resultado está en línea con la literatura existente donde los tiempos fuera del vehículo suelen ser más onerosos que los tiempos de viaje en vehículo (Iseki y Taylor, 2009). La constante modal define la preferencia por una u otra alternativa. Cuando la constante modal se define en la alternativa de servicio actual de bus con trasbordo mide la penalización pura por transferencia o por trasbordo (García-Martínez, 2018). Esta constante modal mide la desutilidad que genera la necesidad de cambiar de vehículo sin tener en cuenta el resto de los elementos del trasbordo (tiempo de caminata, el tiempo de espera para trasbordar, accesibilidad y comodidad en los intercambiadores o estaciones). El valor de este parámetro es negativo lo que significa que para los individuos de la muestra tener que realizar trasbordo les genera una determinada desutilidad. En el caso del modelo 1 esta desutilidad está definida para todos los tipos de trasbordo, para el modelo 2 esta desutilidad está definida según sea un trasbordo urbano, un trasbordo interurbano o un trasbordo interurbano-urbano, mientras que para el modelo 3 esta desutilidad está definida según sea trasbordo urbano o no-urbano. En general, en todos los modelos la menor desutilidad generada por la necesidad de realizar trasbordo es en el caso del trasbordo urbano, esto es, presenta una menor penalización ${ }^{77}$ por la necesidad de trasbordar. En los casos de trasbordo no-urbano, la penalización por trasbordar es mayor.

Tabla 4

Definición de variables para la estimación de los modelos

\begin{tabular}{|c|c|c|c|}
\hline Variables & Definición (unidades) & Parámetro & Signo esperado \\
\hline Tiempo de viaje $(T)$ & $\begin{array}{l}\text { Tiempo total de viaje en bus } \\
\text { (minutos) }\end{array}$ & $\theta_{t}$ & Negativo \\
\hline Coste del viaje $(\mathrm{C})$ & $\begin{array}{l}\text { Coste total del viaje en bus } \\
\text { (céntimos de euro) }\end{array}$ & $\theta_{c}$ & Negativo \\
\hline Frecuencia $(\mathrm{F})$ & $\begin{array}{l}\text { Tiempo entre dos buses } \\
\text { consecutivos (minutos) }\end{array}$ & $\theta_{t e}$ & Negativo \\
\hline Trasbordo Urbano (Dtu) & $\begin{array}{c}\text { Variable ficticia que toma valor } 1 \\
\text { cuando el trasbordo es entre } \\
\text { líneas de Guaguas Municipales, } 0 \\
\text { en otro caso }\end{array}$ & $\theta_{t u}$ & Negativo \\
\hline $\begin{array}{l}\text { Trasbordo Interurbano } \\
\left(D_{\mathrm{ti}}\right)\end{array}$ & $\begin{array}{c}\text { Variable ficticia que toma valor } 1 \\
\text { cuando el trasbordo es entre } \\
\text { líneas de Global, } 0 \text { en otro caso }\end{array}$ & $\theta_{t i}$ & Negativo \\
\hline $\begin{array}{c}\text { Trasbordo Interurbano- } \\
\text { Urbano }\left(D_{\text {tiu }}\right)\end{array}$ & $\begin{array}{c}\text { Variable ficticia que toma valor } 1 \\
\text { cuando el trasbordo es entre } \\
\text { líneas de Guaguas Municipales y } \\
\text { Global, } 0 \text { en otro caso }\end{array}$ & $\theta_{\text {tiu }}$ & Negativo \\
\hline $\begin{array}{c}\text { Trasbordo No Urbano } \\
\left(D_{\text {tnu }}\right)\end{array}$ & $\begin{array}{l}\text { Variable ficticia que toma valor } 1 \\
\text { cuando el trasbordo es entre } \\
\text { líneas de Global y entre líenas de } \\
\text { Glonal y Guaguas Municipales, } 0 \\
\text { en otro caso }\end{array}$ & $\theta_{t n u}$ & Negativo \\
\hline
\end{tabular}

Los modelos 1 y 3 son una versión restringida del modelo 2 que establece constantes modales específicas por tipo de trasbordo (urbano, interurbano e interurbano-urbano), mientras que el modelo 1 es una versión restringida del modelo 3 que especifica constantes modales según el trasbordo sea urbano o no-urbano. Para poder determinar el mejor modelo en términos estadísticos se utiliza el test

\footnotetext{
${ }^{77}$ Un valor en términos absolutos menor de la constante modal.
} 
de razón de verosimilitud (Ortúzar y Willumsen, 2011), que establece como hipótesis nula que el modelo restringido es una especificación correcta con respecto al modelo no restringido. Dicho test tiene la siguiente expresión:

$$
L R=-2\left\{l^{*}\left(\hat{\theta}_{r}\right)-l^{*}(\hat{\theta})\right\}
$$

donde $l^{*}\left(\hat{\theta}_{r}\right)$ es la log-verosimilitud en convergencia del modelo restringido y $l^{*}(\hat{\theta})$ es la del modelo más general. Este test estadístico distribuye asintóticamente $\chi^{2}$ con $\mathrm{r}$ grados de libertad, siendo $\mathrm{r}$ el número de restricciones lineales. La hipótesis nula se rechaza si $L R>X_{r, 95}^{2}$, por lo que el modelo general es aceptado. El modelo 1 es una versión restringida del modelo 2 con tres restricciones lineales, así el valor de LR es 11,4>7,82, así que se acepta el modelo 2, donde la constante modal se especifica por tipo de trasbordo. El modelo 3 es una versión restringida del modelo 2 con dos restricciones lineales, el valor de LR es $0,39<7,82$, por lo que se acepta la hipótesis nula, esto es, el modelo 3 es una especificación correcta. Si se comparan el modelo 1 como modelo restringido del modelo 3 con dos restricciones lineales se obtiene un valor de LR igual a 11,01>5,99 por lo que se rechaza la hipótesis nula y el modelo 3 es una especificación correcta. Por tanto, según el test de razón de verosimilitud y según los contrastes de hipótesis realizados, el modelo 3 con dos parámetros específicos para la constante modal es una mejor especificación en términos estadísticos que los modelos 1 y 2.

Tabla 5

Resultados de las estimaciones

\begin{tabular}{|c|c|c|c|c|}
\hline Variable & Parámetro & Modelo 1 & Modelo 2 & Modelo 3 \\
\hline Constante modal & $\theta$ & $\begin{array}{c}-6,38^{1} \\
(-5,88)^{2}\end{array}$ & - & - \\
\hline $\begin{array}{l}\text { Constante modal } \\
\text { Urbano }\end{array}$ & $\theta t u$ & - & $\begin{array}{c}-4,69 \\
(-4,72)\end{array}$ & $\begin{array}{c}-4,68 \\
(-4,71)\end{array}$ \\
\hline $\begin{array}{l}\text { Constante modal } \\
\text { Interurbano }\end{array}$ & $\theta_{t i}$ & - & $\begin{array}{c}-8,41 \\
(-5,21)\end{array}$ & - \\
\hline $\begin{array}{l}\text { Constante modal } \\
\text { Interurbano-Urbano }\end{array}$ & $\theta_{\text {tiu }}$ & - & $\begin{array}{l}-7,64 \\
(-4,99)\end{array}$ & - \\
\hline $\begin{array}{l}\text { Constante modal } \\
\text { No Urbano }\end{array}$ & $\theta_{\text {tnu }}$ & - & - & $\begin{array}{c}-8.02 \\
(-5.60)\end{array}$ \\
\hline Tiempo de viaje & $\theta_{t}$ & $\begin{array}{l}-0,073 \\
(-4,34)\end{array}$ & $\begin{array}{l}-0,071 \\
(-4,28)\end{array}$ & $\begin{array}{l}-0,069 \\
(-4,23)\end{array}$ \\
\hline Coste del viaje & $\theta_{c}$ & $\begin{array}{l}-0,141 \\
(-8,59)\end{array}$ & $\begin{array}{l}-0,144 \\
(-8,66)\end{array}$ & $\begin{array}{c}-1,44 \\
(-8,65)\end{array}$ \\
\hline Frecuencia & $\theta_{t e}$ & $\begin{array}{l}-0,101 \\
(-4,68)\end{array}$ & $\begin{array}{l}-0,107 \\
(-4,98)\end{array}$ & $\begin{array}{l}-0,105 \\
(-5,11)\end{array}$ \\
\hline Sigma & $\sigma$ & $\begin{array}{c}5,27 \\
(7,26)\end{array}$ & $\begin{array}{c}5,01 \\
(7,58)\end{array}$ & $\begin{array}{c}4,99 \\
(7,81)\end{array}$ \\
\hline $\begin{array}{l}\text { Verosimilitud del } \\
\text { modelo }\end{array}$ & $I^{*}(\theta)$ & $-756,468$ & $-750,768$ & $-750,962$ \\
\hline Rho cuadrado & $\rho^{2}$ & 0,545 & 0,548 & 0,548 \\
\hline Observaciones & & 2416 & 2416 & 2416 \\
\hline Individuos & & 302 & 302 & 302 \\
\hline
\end{tabular}

\section{DISPOSICIONES A PAGAR}

La disposición a pagar (DAP) es una medida de valoración económica por parte de los individuos cuando realizan su elección (Varian, 2011). Según la teoría microeconómica de las elecciones discretas (McFadden, 1981), esta disposición a pagar o valor subjetivo de la característica $\mathrm{k}$ es igual al cociente negativo entre la utilidad marginal del atributo o característica y la utilidad marginal de la renta siendo 

ésta igual a menos la utilidad marginal del coste. Por tanto, tenemos la siguiente expresión para el cálculo de las disposiciones a pagar o valor subjetivo del atributo o característica k:

$$
D A P_{K}=-\frac{\partial V / \partial k}{\partial V / \partial R}=\frac{\partial V / \partial k}{\partial V / \partial C}
$$

En la Tabla 6 se presentan los valores de las disposiciones a pagar por no trasbordar obtenidos para el modelo 3, así como los intervalos de confianza. Los intervalos de confianza son obtenidos siguiendo la propuesta de Armstrong et al. (2001). Se calcula la disposición a pagar por no trasbordar según los dos tipos de trasbordo como el cociente entre el coeficiente estimado para las constantes modales por tipo de trasbordo y el coeficiente de la variable coste del viaje. El resultado obtenido es la disposición a pagar por no trasbordar. En el caso del trasbordo no-urbano, los entrevistados estarían dispuestos a pagar 0,56 euros por no trasbordar, mientras que, en el caso del trasbordo urbano, dicha disposición a pagar por no trasbordar sería menor y de 0,33 euros. Comparando las disposiciones a pagar por no realizar un trasbordo urbano o no-urbano, los individuos de la muestra estarían dispuestos a pagar 1,7 veces más para evitar el trasbordo en el caso de los trasbordos no-urbanos. Este resultado puede estar indicando que, en el caso de los viajes entre distintos municipios, que implican trasbordos no-urbanos, podría estudiarse la posibilidad de definir nuevas líneas siempre que exista una demanda que justifique la creación de esa nueva línea ${ }^{78 .}$

A pesar de que en la literatura hay diferentes estudios sobre trasbordo, la comparación de los resultados puede no ser adecuada dado que los estudios se realizan con distinta metodología y en diferentes contextos de trasbordo. Liu et al. (1997), Wardman et al. (2001) y Currie (2005) indican que la penalización del trasbordo depende del modo de transporte. García-Martínez et al. (2018) obtienen que la penalización pura del trasbordo es diferente cuando el trasbordo se realiza entre diferentes modos. Estos autores obtienen dicha valoración en unidades de tiempo en el vehículo, por tanto, no es posible la comparación con los resultados del presente estudio. Navarrete y Ortúzar (2013) obtienen los valores en unidades monetarias de la penalización del trasbordo. Sin embargo, en el caso de la penalización pura del trasbordo toman como referencia el trasbordo bus-bus por lo que tampoco tenemos datos para comparar. Iseky y Taylor (2009) realizan una extensa revisión de los diferentes estudios sobre trasbordo en el que indican que la valoración económica del trasbordo es muy amplia siendo el menor valor de 0,36-21,6 euros79 y el valor más alto 0,82-49,3 euros. Estos autores indican que la penalización del trasbordo del bus es la más alta en comparación con otros tipos de trasbordos. Además, señalan que el trasbordo varía por modo y ciudad, entendiendo que es necesario el estudio de cada sistema de transporte público para identificar las preferencias de los usuarios. Los resultados obtenidos en este trabajo para los distintos tipos de trasbordo se encuentran entre los valores recogidos en la literatura.

Tabla 6

Disposiciones a pagar

\begin{tabular}{|l|c|c|}
\hline Disposiciones a pagar (DAP) & Valor obtenido & $\begin{array}{c}\text { Intervalo de } \\
\text { confianza }\end{array}$ \\
\cline { 2 - 4 } & (Euros/viaje) & (Euros/viaje) \\
\hline DAP por no trasbordar Urbano & $0,33^{1}$ & {$\left[0,20^{2}-0,46^{3}\right]$} \\
\hline DAP por no trasbordar No Urbano & 0,56 & {$[0,39-0,73]$} \\
\hline \\
$\begin{array}{l}{ }^{1} \text { En euros } \\
{ }^{2} \text { Límite inferior del intervalo de confianza }\end{array}$
\end{tabular}

\footnotetext{
${ }^{78}$ La empresa Global ha creado nuevas líneas entre diferentes municipios que suponen la eliminación de trasbordo interurbano.

${ }^{79}$ El tipo de cambio dólar euro en 2009 es 1,40.
} 


\section{CONCLUSIONES}

Los sistemas integrados de transporte pretenden ofertar un servicio de transporte público que permita acceder al mayor número de destinos por parte de los usuarios. Este servicio de transporte público no siempre puede ofertarse como un servicio directo entre origen y destino por lo que el trasbordo se convierte en un elemento clave del propio sistema, dado que implica aumento en los tiempos de desplazamientos, así como incremento en el coste de dicho desplazamiento si no existe integración tarifaria como en el caso estudiado en este artículo.

En este trabajo se estudia la valoración de la penalización pura del trasbordo para los usuarios de transporte público en la isla de Gran Canaria. Para ello se realiza un experimento de PD que consiste en un juego de elección entre dos alternativas de bus: el servicio actual de bus con trasbordo y un servicio hipotético de bus directo. A cada entrevistado se le presentaron 8 juegos de elección con las variables seleccionadas. Dado que el servicio de transporte público está ofertado por dos empresas se consideraron tres tipos de trasbordo: trasbordo urbano entre líneas de la empresa que provee el servicio en la ciudad de Las Palmas de Gran canaria (102 entrevistados), trasbordo interurbano entre las líneas de la empresa que provee el servicio entre los municipios de la isla y la capital (99 entrevistados) y trasbordo interubano-urbano entre líneas de las dos empresas (101 entrevistados). La muestra total está compuesta por 302 individuos y 2416 observaciones.

Se estimaron modelos ML con error homocedástico definiendo la constante modal en la alternativa de servicio actual de bus con trasbordo para medir la penalización pura del trasbordo (García-Martínez, 2018). Los valores de las disposiciones a pagar por no trasbordar son obtenidos por tipo de trasbordo, siendo el valor más alto en el caso del trasbordo no-urbano mientras que el valor más bajo se obtiene para el trasbordo urbano.

Los resultados pueden indicar la necesidad de despenalizar el trasbordo eliminando el pago del segundo trayecto en unas determinadas condiciones para así fomentar el uso del transporte público. Concretamente, estos valores fueron utilizados para realizar un análisis coste-beneficio de diferentes propuestas para fomentar el uso del transporte público que incluían la despenalización del trasbordo.

El trabajo realizado aporta evidencia empírica sobre la penalización pura del trasbordo obteniendo una valoración económica de dicha penalización que puede ser tenida en cuenta por las instituciones públicas para el diseño de políticas de fomento del transporte público. Además, el estudio se centra en la valoración del servicio de transporte público desde la perspectiva de los usuarios de transporte y en este contexto, el trabajo puede aportar soluciones en la toma de decisiones de la autoridad responsable especialmente en la definición de los criterios para las concesiones públicas del sistema de transporte público.

No obstante, este trabajo presenta algunas limitaciones. En primer lugar, que el experimento de PD se centra en sólo tres atributos modales y no se incluyeron otros aspectos relevantes del trasbordo (confort, seguridad, instalaciones, entre otros). Esto se debe a que el grupo focal para el diseño del experimento de PD se centra en la alternativa de servicio hipotético de bus directo. En este sentido, trabajar en la definición de conceptos nuevos sobre los distintos elementos que definen el trasbordo según la literatura existente sería una línea interesante de investigación futura. En segundo lugar, que el estudio se centra en los usuarios de transporte público y podría ser interesante conocer también las preferencias de los usuarios potenciales. Para ello sería interesante la combinación de datos de Preferencias Reveladas con datos de PD aportando un valor añadido la combinación de los dos tipos de datos. Finalmente, estas limitaciones serán tenidas en cuenta para futuros trabajos de investigación.

\section{AGRADECIMIENTOS}

La autora agradece la financiación por parte del Cabildo de Gran Canaria y a las empresas de transporte Guaguas Municipales y Global por facilitar toda la información necesaria para la realización del estudio, así como su colaboración en las fases previas del estudio.

La autora también agradece los comentarios de los dos evaluadores que han contribuido a la mejora del artículo. Cualquier error u omisión es responsabilidad de la autora. 
AMADOR, F.J., GONZÁLEZ, R.M. y ORTÚZAR, J. de D (2005): "Preference Heterogeneity and willingness to pay for travel time savings" en Transportation, 32: pp. 627-647.

AMADOR, F.J., y GONZÁLEZ, R.M. (2005): "El valor subjetivo del tiempo de viaje de los estudiantes universitarios cuando las preferencias son heterogéneas" en Hacienda Pública Española, 174: pp. 25 - 42.

ARMSTRONG P, GARRIDO R y ORTÚZAR J. de D. (2001): "Confidence intervals to bound the value of time" en Transportation Research E, 37: pp. 143-161.

ASENSIO, J, y MATAS, A. (2006): "Valoración del tiempo y la fiabilidad: La C-32 en Barcelona, memoria de proyecto de investigación Costes, precios y externalidades para las infraestructuras de transporte" en Memoria de proyecto de investigación Costes, precios y externalidades para las infraestructuras de transporte, Programa Nacional de I+D en Transporte del Ministerio de Fomento (2002-2005).

BARRIOS, M.C. y MARTíNEZ, M. A. (1999): "El valor del tiempo en los desplazamientos al trabajo: una estimación en el caso de Cádiz" en Estudios de Economía Aplicada, 12: pp. 5-16.

BIERLAIRE, M. (2003): "A free package for the estimation of discrete choice models" en Proceedings of the 3rd Swiss Transportation Research Conference.

BLIEMER, M. C. y JOHN M. ROSE, (2005): "Efficiency and sample size requirements for stated choice studies" en Institute of Transport Studies and Logistics, Working Paper ITLS-WP-05-08.

BLIEMER, M.C.J. y ROSE, J.M. (2010): "Construction of experimental designs for mixed logit models allowing for correlation across choice observations” en Transportation Research Part B: Methodological, 44(6): pp.720734.

BRADLEY, M. A. y KROES, E. (1990): "Forecasting issues in stated preference survey research" en 69th TBR Annual Meeting, Washington DC, Enero 1990, EE.UU.

CANTOS, P, y ÁLVAREZ, O. (2006): "El valor del tiempo y la congestión en los accesos en las grandes ciudades: El caso de la radial-3 y la autovía A-3 en Madrid" en Memoria de proyecto de investigación Costes, precios y externalidades para las infraestructuras de transporte, Programa Nacional de I+D en Transporte del Ministerio de Fomento (2002-2005).

CASCAJO, R.; LOPEZ, E.; HERRERO, F. y MONZON, A. (2018): "User perception of transfers in multimodal urban trips: A qualitative study" en International Journal of Sustainable Transportation, 0: pp. 1-14.

CHOWDHURY, S. y CEDER, A. (2016): 'Users' willingness to ride an integrated public-transport service: A literature review" en Transport Policy, 48: pp. 183-195.

CURRIE, G. (2005): "The Demand Performance of Bus Rapid Transit” en Journal of Public Transportation, 8(1): pp. 41-55.

DELL'OLIO, L.; IBEAS, A. y CECIN, P. (2011): "The quality of service desired by public transport users" en Transport Policy, 18(1): pp.217-227.

DOMENCICH, T. A. y MCFADDEN, D. (1975). Urban Travel Demand. A Behavioral Analysis. Amsterdam: North Holland Publishing Co.

DOUGLAS, N.J. y JONES, M. (2013): "Estimating transfer penalties and standardized income values of time by Stated Preference Survey" en 36th Australian Transport Resarch Forum, Brisbane, pp. 1-21.

ESPINO, R., (2003): "Análisis y predicción de la demanda de transporte de pasajeros. Una aplicación al estudio de dos corredores de transporte en Gran Canaria", tesis doctoral, Universidad de Las Palmas de Gran Canaria.

ESPINO, R., ORTÚZAR, J. de D., y ROMÁN, C. (2004): "Diseño de preferencias declaradas para analizar la demanda de viajes" en Estudios de Economía Aplicada, 22-3: pp. 759-793.

ESPINO, R., ORTÚZAR, J. de D., y ROMÁN, C. (2007): "Understanding suburban travel demand: Flexible modelling with revealed and stated choice data" en Transportation Research Part A: Policy and Practice, 41(10): pp. 899-912.

ESPINO, R., ROMÁN, C., y ORTÚZAR, J. de D. (2006): "Analysing demand for suburban trips: A mixed RP/SP model with latent variables and interaction effects" en Transportation, 33(3): pp. 241-261.

GARCÍA-MARTÍNEZ (2018): "Modelización de la penalización del transbordo en transporte público. Un enfoque cualitativo y cuantitativo", tesis doctoral, Universidad Polítécnica de Madrid.

GARCIA-MARTINEZ, A.; CASCAJO, R.; JARA-DIAZ, S. R.; CHOWDHURY, S. y MONZON, A. (2018): "Transfer penalties in multimodal public transport networks" en Transportation Research Part A: Policy and Practice, 114 (January): pp.52-66.

GSCHWENDER, A.; JARA-DÍAZ, S. y BRAVO, C. (2016): "Feeder-trunk or direct lines? Economies of density, transfer costs and transit structure in an urban context" en Transportation Research Part A: Policy and Practice, 88: pp. 209-222.

GUO, Z. y WILSON, N. H. M. (2004): "Assessment of the Transfer Penalty for Transit Trips Geographic Information System-Based Disaggregate Modeling Approach" en Transportation Research Record: Journal of the Transportation Research Board, 1872: pp. 10-18.

GUO, Z. y WILSON, N. H. M. (2011): "Assessing the cost of transfer inconvenience in public transport systems: A case study of the London Underground" en Transportation Research Part A: Policy and Practice, 45(2): pp. 91-104.

ISEKI, H. y TAYLOR, B. D.(2009): "Not All Transfers Are Created Equal: Towards a Framework Relating Transfer Connectivity to Travel Behaviour" en Transport Reviews, 29: pp.(777-800).

ISTAC (2016): Instituto Canario de Estadística del Gobierno de Canarias. 
LIU, R.; PENDYALA, R. M.; y POLZIN, S. (1997): "Assessment of Intermodal Transfer Penalties Using Stated Preference Data" en Transportation Research Record, 1607 (971380): pp. 74-80.

LOUVIERE, J. J.; HENSHER, D. A. y SWAIT, J. D. (2000): Stated Choice Methods: Analysis and Application. Cambridge: Cambridge University Press.

MATAS, A. (1991): "La demanda de transporte urbano: Un análisis de las elasticidades y valoraciones del tiempo" en Investigaciones Económicas, vol. XV, n² 2: pp. 249-267.

MCFADDEN, D. (1981). "Econometric Models of Probabilistic Choice" en C. Manski y D. Mcfadden (Eds.): Structural Analysis of Discrete Choice Data (pp. 198-272). Cambridge: MIT Press.

MCFADDEN, D. y TRAIN, K. (2000): "Mixed MNL Models for Discrete Response" en Journal of Applied Econometrics, 15: pp. 447-470.

NAVARRETE, F. J. y ORTÚZAR, J. de D. (2013): "Subjective valuation of the transit transfer experience: The case of Santiago de Chile" en Transport Policy, 25: pp. 138-147.

ORTÚZAR, J. de D. y WILLUMSEN, L. (2011). Modelling Transport (4th ed.). Chichester: John Wiley \& Sons, Ltd.

OSUNA, E.E. y NEWELL, G.F. (1972): "Central strategies for an idealized public transport system" en Transportation Science, 6 (1), pp. 52-72.

PAULLEY, N., BALCOMBE, R., MACKETT, R., TITHERIDGE, H., PRESTON, J., WARDMAN, M., JEREMY, S. y WHITE, P. (2006): "The demand for public transport: The effects of fares, quality of service, income and car ownership" en Transport Policy, 13(4): pp. (295-306).

RAND EUROPE (2001). WinMINT 2.1 User Manual.

RAVEAU, S.; GUO, Z.; MUÑOZ, J. C. y WILSON, N. H. M. (2014): "A behavioural comparison of route choice on metro networks: Time, transfers, crowding, topology and socio-demographics" en Transportation Research Part A: Policy and Practice, 66(1), pp. 185-195.

REVELT, D. y TRAIN, K. E. (1998): "Mixed Logit with Repeated Choices" en Review of Economics and Statistics, 80(4): pp. 647-657.

ROMÁN, C., MARTíN, J. C., ESPINO, R., CHERCHI, E., ORTÚZAR, J. de D., RIZZI, L. I., GONZÁLEZ, R.M. y AMADOR, F. J. (2014a): "Valuation of travel time savings for intercity travel: The Madrid-Barcelona corridor" en Transport Policy, 36: pp. 105-117.

ROMÁN, C.; MARTíN, J. C. y ESPINO, R. (2014b): "Using Stated Preferences to Analyse the Service Quality of Public Transport" en International Journal of Sustainable Transportation, 8 (1): pp. 28-46.

ROMÁN, C.; MARTíN, J.C.; ESPINO, R. y ARENCIBIA, A.I. (2012): "Efficient Versus Non-Efficient Stated Choice Designs. A Comparison in A Mode Choice Context" en Scienze Regionali - Italian Journal of Regional Science, 11(3): pp. 31-50.

SCHAKENBOS, R.; PAIX, L.L.; NIJENSTEIN, S. y GEURS, K.T. (2016): "Valuation of a transfer in a multimodal public transport trip" en Transport Policy, 46: pp. 72-81.

TRAIN, K.E. (2009): Discrete Choice Methods with simulation (2nd ed.). Cambridge: University Press.

VARIAN, H.R. (2011): Microeconomía intermedia (8 ed.). Barcelona: Antoni Bosch.

WARDMAN, M.; HINE, J., y STRADLING, S. (2001): "Interchange and Travel Choice" Volume 1 en Edinburgh: Scottish Executive Central Research Unit, 1.

WARDMAN, M. y HINE, J. (2000): Costs of Interchange: A review of the Literature, ITS-WP-546. 